\title{
MISE EN PLACE DES PREMIERS COMPORTEMENTS ET UTILISATION DE L'HABITAT APRÈS L'ÉMERGENCE CHEZ LES SALMONIDÉS D'EAU COURANTE.
}

\author{
M. HELAND (1), P. GAUDIN (2), A. BARDONNET (3)
}

(1) INRA, Station d'Hydrobiologie. Unité Ecologie des Poissons, BP 3, 64310 Saint-Péesur-Nivelle, France.

(2) Laboratoire de Biologie animale et Ecologie, URA CNRS 1974, Université Cl. Bernard Lyon I, 43 bd du 11 Novembre 1918, 69622 Villeurbanne Cedex, France.

(3) INRA, Laboratoire d'Ecologie aquatique, 65 rue de St Brieuc, 35042 Rennes Cedex, France.

\section{RÉSUMÉ}

Chez les salmonidés rhéophiles, la courte période de quelques jours qui suit l'émergence hors des graviers de la frayère a une importance déterminante pour la survie individuelle. Qu'il soit en situation d'allopatrie ou de sympatrie, l'alevin doit sélectionner un habitat lui permettant de ne pas être déplacé vers l'aval par le courant, de capturer des proies et d'échapper à la prédation.

Les travaux que nous avons réalisés ces dernières années sur différentes espèces comme la Truite commune (Salmo trutta), le Saumon atlantique (Salmo salar) ou l'Ombre commun (Thymallus thymallus) illustrent la variabilité des comportements de postémergence, en réponse à des conditions environnementales diversifiées. Différentes formes de dispersion apparaissent en direction de l'aval ou des zones de bordure, ou encore à proximité immédiate de la zone de frayère vers des microhabitats plus ou moins benthiques adaptés à la taille et aux capacités de nage des différentes espèces. La présence de congénères influence cette microrépartition par compétition pour les meilleurs postes d'accès à la nourriture (dérive d'invertébrés). L'existence de prédateurs lors de l'émergence peut entraîner le retardement de celle-ci ou des réenfouissements dans les graviers après émergence, mais aussi une augmentation de la dévalaison.

\section{FIRST BEHAVIOURAL ADJUSTMENTS IN RELATION TO HABITAT USE AFTER EMERGENCE IN RUNNING WATER SALMONIDS.}

\section{SUMMARY}

In rheotactic salmonids, the short period (a few days) after emergence from the gravel is critically important for individual survival. In allopatric or sympatric situations, fry have to select an habitat providing shelters against predators or downstream displacement by water current, or food as invertebrates drift.

Experiments done during the last few years with different salmonid species such as Brown trout (Salmo trutta), Atlantic salmon (Salmo salar) and Grayling (Thymallus thymallus) showed post-emergent behavioural variability corresponding to varied environmental conditions. Different forms of fry dispersal appeared through downstream movement or displacement towards the banks and/or in the vicinity of the redd area, to more or less benthic microhabitats well adjusted to the size and swimming capacity of the different species. The presence of conspecifics influenced microdistribution through competition for the best stations for prey capture (invertebrates drift). The presence of predators could delay emergence or cause reburial of fry in the gravel, but more often increase downstream movement. 


\section{INTRODUCTION}

Chez les Salmonidés d'eau courante, la sortie des graviers de la frayere correspond a une étape essentielle du cycle vital comparable à une véritable "métamorphose" (BALON. 1984). Cette sortie constitue le point de départ d'un processus de positionnement de l'animal dans un milieu nouveau, très différent des conditions de vie dans la frayère. L'alevin émergeant doit s'adapter rapidement pour se maintenir ou se déplacer dans le courant, rechercher la nourriture, synchroniser son rythme d'activité. éviter les prédateurs. En fonction de ces différents ajustements, il "choisit" un habitat particulier à partir duquel il s'intègre dans l'écosystème.

Cette phase de l'émergence et des premiers instants de la vie en eau libre, caractérisée par des mortalités très importantes, est fondamentale pour le devenir d'une cohorte d'alevins issus d'une même frayère et, par conséquent, pour la survie et le développement des populations (HELAND, 1991 : ELLIOTT, 1994). En raison des difficultés techniques d'échantillonnage et d'observation, les connaissances sur cette période de mise en place des premiers comportements présentent encore des lacunes et justifient l'actualité des recherches sur les jeunes stades conduites par différentes équipes en Europe et en Amérique du Nord. Une tentative de synthèse des connaissances acquises est présentée ici en soulignant les résultats récents sur le sujet. Les exemples sont pris chez les Salmonidés d'eau courante tels que la Truite (Salmo trutta L.). le Saumon (Salmo salar L.) et l'Ombre commun (Thymallus thymallus L.).

Après quelques rappels sur les modalités de l'émergence, le placement de l'alevin dans l'habitat sera examiné tout d'abord d'un point de vue général puis en fonction des différents facteurs écologiques qui peuvent l'influencer.

\section{L'ÉMERGENCE}

La frayère constitue le premier habitat des salmonides d'eau courante. Son emplacement, choisi par les géniteurs, se situe naturellement dans une zone favorable aux besoins physiologiques de développement des larves et alevins notamment en ce qui concerne la température, l'oxygène et la qualité de l'eau. Dès sa sortie des graviers, l'alevin se trouve ainsi dans une zone à l'intérieur ou à proximité de laquelle il peut trouver le microhabitat correspondant à ses aptitudes sensori-motrices d'utilisation de l'environnement.

Ce point de départ - la zone de frayère - est assez semblable chez les différentes espèces de Salmonidés : tête de courant rapide où l'eau peut circuler par percolation à travers un substrat grossier, mélange de graviers et galets (tête de seuil en fin de mouille selon la nomenclature de MALAVOI, 1989). Cependant, de petites différences existent entre les frayères de saumons sur des courants plus rapides et plus vastes présentant une granulométrie plus grosse et les frayères de truites sédentaires ou d'ombres communs. Ces différences s'estompent avec les frayères de truites de mer comparables en taille aux saumons, ainsi qu'avec les frayères de truites de lac (MELHAOUI, 1985). Cependant, ces différences spatiales interspécifiques minimes s'accompagnent d'une importante variabilité temporelle puisque l'ombre commun fraye au printemps, alors que les 2 autres espèces se reproduisent en fin d'automne-début d'hiver, dans l'hémisphère Nord.

L'émergence elle-même, qui correspond à la sortie des alevins hors des graviers, reste un phénomène délicat à caractériser dans le temps. Contrairement à l'éclosion, quasi synchrone pour les oeufs d'une même ponte dans des conditions relativement stables de température, elle s'étale sur plusieurs jours, voire plusieurs semaines. Elle correspond à un état physiologique de l'alevin qui quitte le compartiment sous gravier au moment où ses réserves vitellines sont proches de l'épuisement. Les premières prises alimentaires sont contemporaines de l'émergence chez la truite et le saumon même si la vésicule vitelline n'est pas totalement résorbée. En terme de développement morphologique, l'émergence peut se réaliser alors que l'oesophage est encore obstrué par un bouchon muqueux et donc avant les premières prises alimentaires comme on l'observe chez l'ombre commun (BARDONNET et al., 1993 a), ou, au contraire, après la disparition de ce bouchon comme chez le saumon dont l'estomac peut contenir des limons (VIGNES et HELAND, 1995, ce numéro). 
Des travaux expérimentaux récents ont permis de différencier l'émergence proprement dite (sortie des graviers) des premiers déplacements des alevins (BARDONNET et al., 1993 b). Globalement, l'émergence se produit la nuit au même moment que les premiers déplacements chez les espèces du genre Salmo, comme chez la plupart des Salmonidés. Par contre, l'ombre commun émerge plutôt le matin et diffère ses premiers déplacements vers l'aval la nuit suivante (BARDONNET et GAUDIN, 1990). Ces activités sont essentiellement synchronisées par les variations d'intensité lumineuse au cours de l'alternance jour-nuit. La température intervient également, soit comme synchronisateur, en synergie avec la lumière (BARDONNET et GAUDIN, 1991), soit comme facteur d'influence sur le métabolisme des larves en retardant (température basse) ou favorisant (température optimale) l'enchaînement comportemental de l'émergence (CALLEJA-BOVEDA, 1987). L'émergence s'effectue par une sortie des graviers plus ou moins nette selon l'espèce et les conditions physiques (granulométrie, débit...). Chez l'ombre commun, où elle présente le caractère le plus définitif, de fréquents réenfouissements sont observés lorsque la granulométrie est grossière et homogène contrairement à ce qui est observé en granulométrie hétérogène (BARDONNET, 1989). Dans le genre Salmo, certains individus peuvent sortir furtivement et se réenfouir pour ressortir plus tard alors que d'autres peuvent "jaillir" des graviers et partir en pleine eau en mouvements rapides et désordonnés pour se poser sur le fond à quelque distance (GAUDIN et HELAND, 1995, ce numéro). Les espaces intra-graviers peuvent également constituer un milieu refuge, notamment lors des perturbations hydrauliques, en particulier pour les espèces qui sont ultérieurement très inféodées au substrat comme le Saumon atlantique. Chez cette dernière espèce, le compartiment sous graviers est utilisé fréquemment pour les déplacements avant l'émergence ou après réenfouissement (BARDONNET, données non publiées). En définitive, l'émergence au sens strict, considérée souvent comme un comportement stéréotypé de - sortie-prise d'air-première alimentation - apparaît davantage comme un phénomène modulable en fonction des facteurs du milieu, cependant sous l'influence majeure du besoin nutritionnel.

\section{LE PLACEMENT DANS L'HABITAT}

A la sortie des graviers de la frayère, l'alevin est confronté à 2 problèmes essentiels : se maintenir contre le courant d'eau en réponse au comportement rhéophile des salmonidés et capturer les proies de la dérive (HELAND, 1991). L'orientation par rapport au courant et la dérive d'invertébrés conduit les alevins à rechercher dès l'émergence des emplacements favorables, en général dans le remous situé à l'aval d'un caillou, d'où ils pourront minimiser leurs dépenses d'énergie sous forme de nage contre le courant et maximiser leurs gains d'énergie par capture des proies véhiculées par le courant proche (fig. 1). Ultérieurement, après une période d'attachement au site par renforcement alimentaire, la stabilisation territoriale intervient comme résultante des compétitions entre alevins pour la possession des bons postes de chasse (HELAND, 1991).

Dès l'émergence, certains individus ne vont pas se maintenir dans la même zone en luttant contre le courant mais vont partir vers l'aval au cours des nuits suivantes. Ils colonisent ainsi progressivement l'aval du ruisseau pauvre en frayères (BEALL et al., 1994). Ces alevins, dévalant dès l'émergence ou immédiatement après, ont la même taille et le même comportement alimentaire que les sédentaires (VIGNES et HELAND, 1993), mais adoptent la nage stationnaire face au courant plus tardivement (HELAND, 1980). La présence de congénères émergés plus tôt influence probablement cette dévalaison massive précoce.

Les différences entre les microhabitats occupés par les alevins dès l'émergence ou après la dévalaison peuvent s'analyser en prenant en considération la spécialisation de chacune des espèces dans l'utilisation de l'habitat. II ressort notamment une certaine gradation par rapport à la vitesse du courant et la proximité du substrat, dans le sens saumon-truite-ombre : les alevins de saumon plus benthiques, mais aussi plus aptes aux déplacements en courant très rapide, se retrouvent dans des zones peu profondes à courant vif $(20$ à $40 \mathrm{~cm} / \mathrm{s})$ et substrat assez grossier (HELAND et DUMAS, 1994). Les alevins de truite occupent des zones peu profondes $(10$ à $40 \mathrm{~cm})$ à courant modéré $(0$ à $20 \mathrm{~cm} / \mathrm{s})$ et substrat de graviers et galets. Ils adoptent davantage une activité de nage stationnaire en 


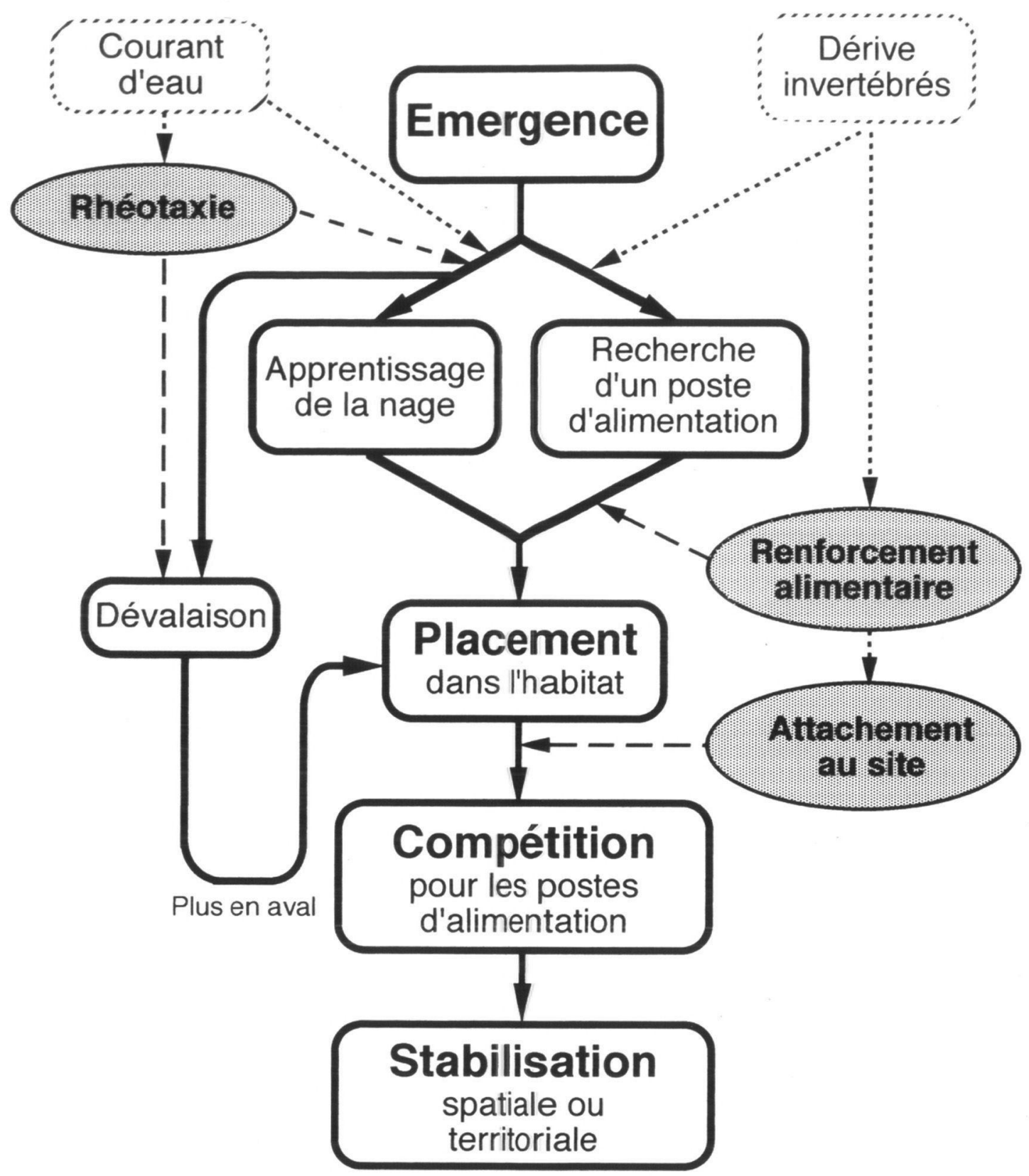

Figure 1 : Représentation simplifiée de la séquence comportementale qui conduit l'alevin de Salmonidés de l'émergence à la stabilisation territoriale . Influence des principaux facteurs externes et des mécanismes internes $\bigcirc-$ en interaction au cours de la séquence.

Figure 1 : Simplified representation of behavioural sequence from emergence to territorial stabilization in Salmonids fry factors in interaction during the sequence.

pleine eau, moins inféodée au substrat que les saumons (GAUDIN et HELAND, 1995, ce numéro). Les alevins d'ombre, plus pélagiques pendant cette première dispersion, se regroupent dans des habitats à courant plus lent (inférieur à $20 \mathrm{~cm} / \mathrm{s}$ ) situés à proximité des rives sur fond de sable ou de limon où ils exploitent en nage stationnaire ou en déplacements erratiques la partie haute de la colonne d'eau sans nécessité de disposer 
d'un obstacle sur le substrat pour lutter contre le courant. Quelques semaines plus tard, ils gagneront le courant principal, un peu plus rapide et plus profond (BARDONNET et al., 1991 ; SEMPESKI et GAUDIN, 1995).

La séquence comportementale qui conduit à l'utilisation du premier habitat après l'émergence, est modulée par différents facteurs abiotiques et biotiques : température, lumière et qualité de l'eau, évoqués plus haut mais aussi caractéristiques morphodynamiques et présence de congénères ou autres espèces. Les caractéristiques morphodynamiques, vitesse-hauteur d'eau-substrat, ont fait l'objet d'attentions particulières pour la caractérisation de l'habitat et sa modélisation. Cependant, peu de travaux portent sur les très jeunes stades de salmonidés, pendant les quelques jours qui suivent l'émergence. Chez l'ombre commun, une description détaillée de l'utilisation de l'habitat a permis de démontrer l'importance de l'alternance jour-nuit : les alevins se rapprochent des berges pendant la nuit et se reposent sur le fond dans quelques centimètres d'eau (SEMPESKI et GAUDIN, 1995). Par la suite, lorsque les alevins ont gagné le courant principal, ce comportement de retour nocturne vers les berges se maintient non seulement la nuit, mais également lors des crues artificielles (VALENTIN et al., 1994).

La présence de congénères de la même classe d'âge est un élément essentiel du schéma de colonisation de l'habitat dès la sortie des graviers (GAUDIN et HELAND, 1995, ce numéro). L'ajustement de la densité des alevins aux ressources spatiales et trophiques du milieu, se construit à travers une série d'interactions de type apprentissage ou de type agressivité qui pourra aboutir ultérieurement au modèle territorial. La présence de congénères plus âgés, d'espèces d'accompagnement ou d'espèces concurrentes, tous prédateurs potentiels, aboutit à des réactions d'évitement soit par retard d'émergence, soit par augmentation de la dévalaison comme cela a été démontré chez la truite ou le saumon atlantique (GAUDIN et HELAND, 1984 ; BARDONNET et HELAND, 1994 ; BEALL et al., 1989). L'utilisation de microhabitats légèrement différents pendant cette phase critique, permet d'éviter la confrontation directe entre espèces ou classes d'âge de la même espèce (HELAND et DUMAS, 1994).

\section{CONCLUSION}

En définitive, la séquence comportementale d'émergence et d'exploitation du premier habitat apparaît comme un phénomène d'une grande flexibilité dans le temps, de même que dans l'espace par le biais de la dévalaison. Cette flexibilité permet un ajustement aux conditions environnementales, que ce soient les crues ou les prédateurs. Cet évitement préfigure les réactions de ségrégation interactive observées entre espèces sympatriques de Salmonidés conduisant chaque espèce à sélectionner le microhabitat le mieux adapté à ses aptitudes particulières, nécessairement différent de celui utilisé par l'espèce concurrente (HEARN, 1987). Les résultats récents rapportés ici soulignent la plasticité comportementale d'utilisation du premier habitat qui s'accorde bien à la nécessité d'adaptation rapide de l'alevin à un environnement très fluctuant. La prise en compte de cette plasticité en terme de variabilité quantifiée au niveau des modèles d'habitat paraît indispensable à leur validation.

\section{BIBLIOGRAPHIE}

BALON E.K., 1984. Reflections on some decisive events in the early life of fishes. Trans. Am. Fish. Soc., 113, 178-185.

BARDONNET A.,1989. Occupation de l'espace par les jeunes stades de Salmonidés : vie sous graviers, émergence et dévalaison chez l'ombre commun, (Thymallus thymallus L., 1758). Thèse de Doctorat de I'Université de Lyon I, 200 p., Lyon Library, microfiche (89/LY01/0137).

BARDONNET A., GAUDIN P., 1990. Diel pattern of downstream post-emergence displacement of grayling (Thymallus thymallus L., 1758). J. Fish Biol., 37, 623-627. 
BARDONNET A., GAUDIN P., 1991. Influence of light and temperature on the emergence rhythm of grayling fry (Thymallus thymallus L., 1758). Can. J. Fish. Aquat. Sci., 48 (7), 1176-1180.

BARDONNET A., GAUDIN P., PERSAT H., 1991. Microhabitat and downstream displacement in young grayling (Thymallus thymallus). Fresh. Biol., 26, 365-376.

BARDONNET A., GAUDIN P., GROLET O., THORPE J., 1993 a. Presence of an oesophageal plug at the time of emergence in grayling (Thymallus thymallus L.) and Atlantic salmon (Salmo salar L.). J. Fish Biol., 43, 500-502.

BARDONNET A., GAUDIN P., THORPE J., 1993 b. Diel rhythm of emergence and of first displacement downstream in trout (Salmo trutta L.), Atlantic salmon (Salmo salar L.) and grayling (Thymallus thymallus L.). J. Fish Biol., 43, 755-762.

BARDONNET A., HELAND M., 1994. The influence of potential predators on the habitat preferenda of emerging brown trout. J. Fish Biol., 45, suppl. A, 131-142.

BEALL E., HELAND M., MARTY C., 1989. Interspecific relationships between emerging Atlantic salmon, Salmo salar, and coho salmon, Oncorhynchus kisutch, juveniles. $J$. Fish Biol., 35, suppl. A, 285-293.

BEALL E., DUMAS J., CLAIREAUX D., BARRIERE L., MARTY C., 1994. Dispersal patterns and survival of Atlantic salmon (Salmo salar L.) juveniles in a nursery stream. ICES J. Mar. Sci., 51, 1-9.

CALLEJA-BOVEDA P., 1987. Influence de la température et de la photopériode sur le développement des activités chez l'alevin de truite commune, Salmo trutta L., en aquarium. DEA Ecologie expérimentale, Univ. Pau et Pays Adour, $43 \mathrm{p}$.

ELLIOTT J.M., 1994. Quantitative ecology and the brown trout. Oxford Univ. Press, Oxford, $286 \mathrm{p.}$

GAUDIN P., HELAND M., 1984. Influence d'adultes de chabots (Cottus gobio L.) sur des alevins de truite commune (Salmo trutta L.) : étude expérimentale en milieux seminaturels. Acta Oecologica, Oecol. applic., 5, 71-83.

GAUDIN P., HELAND M., 1995. Stratégies d'utilisation de I'habitat par les alevins postémergents de truite commune (Salmo trutta) et de saumon atlantique (Salmo salar). Bull. Fr. Pêche Piscic., 337/338/339.

HEARN W.E., 1987. Interspecific competition and habitat segregation among streamdwelling trout and salmon : a review. Fisheries, 12, 24-31.

HELAND M., 1980. La dévalaison des alevins de truite commune, Salmo trutta L. II - Activité des alevins "dévalants" comparés aux sédentaires. Ann. Limnol., 16 (3), 247-254.

HELAND M., 1991. Organisation sociale et territorialité chez la truite commune immature au cours de I'ontogenèse. In : La Truite. Biologie et écologie, 121-149, Baglinière J.L., Maisse G. (Eds), INRA Editions, Paris.

HELAND M., DUMAS J., 1994. Ecologie et comportement des juvéniles. In : Le saumon atlantique, biologie et gestion de la ressource, 29-46, Gueguen J.C., Prouzet P. (Eds), IFREMER, Brest.

MALAVOI J.R., 1989. Typologie des faciès d'écoulement ou unités morphodynamiques des cours d'eau à haute énergie. Bull. Fr. Pêche Piscic., 315, 189-210.

MELHAOUI M., 1985. Eléments d'écologie de la truite de lac (Salmo trutta L.) du Léman dans le système lac-affluent. Thèse 3ème cycle Biol. anim., Univ. Pierre et Marie Curie, Paris VI, $127 \mathrm{p}$.

SEMPESKI P., GAUDIN P., 1995. Habitat selection by grayling. II - Preliminary results on larval and juveniles day time habitats. J. Fish Biol., 47, 2, 345-349. 
VALENTIN S., SEMPESKI P., SOUCHON Y., GAUDIN P., 1994. Short-term habitat use by young grayling, Thymallus thymallus $L$., under variable flow conditions in an experimental stream. Fish. Mgmt. Ecol., 1, 57-65.

VIGNES J.C., HELAND M., 1993. Essai de caractérisation des alevins dévalants : comparaison de leur comportement alimentaire avec celui d'alevins sédentaires chez le saumon atlantique, Salmo salar, en ruisseau semi-naturel. In : Rapp. PIR 2ème année, 21-23, Souchon Y., Gaudin P. (Eds), Univ. Cl. Bernard et CEMAGREF Lyon.

VIGNES J.C., HELAND M., 1995. Comportement alimentaire au cours du changement d'habitat lié à l'émergence chez le saumon atlantique, Salmo salar L., et la truite commune, Salmo trutta L., en conditions semi-naturelles. Bull. Fr. Pêche Piscic., $337 / 338 / 339$. 